Fifth Meeting, March 11th, 1898.

Dr Morgan, Vice-President, in the Chair.

An Analysis of all the Inconclusive Votes possible with 15 Electors and 3 Candidates.

By Professor Steggall.

\title{
A Suggestion for a Shortened Table of Five-Figure Logarithms.
}

By Professor Stgggall.

Note on the Centre of Gravity of a Circular Arc.

By John Dougall, M.A.

Mr Crawford's note on this subject, read at a recent meeting, reminds me of a method $\mathrm{I}$ gave to a class four or five years ago.

\section{Figure 14.}

Let $A M B$ be an arc subtending an angle $2 a$ at the centre $O$ of a circle of radius $a$. The centre of gravity $G_{1}$ lies, from symmetry, on $\mathrm{OM}$ the line from $\mathrm{O}$ to the mid-point of the arc.

Let $\mathrm{G}_{2}$ be the C.G. of an adjacent arc BNC of angle $2 \beta$.

If $\mathrm{G}$ be the C.G. of the whole arc AMBNC, the angle AOG is $a+\beta$.

Thus $\angle \mathrm{G}_{1} \mathrm{OG}=\beta$ and $\angle \mathrm{G}_{2} \mathrm{OG}=a$. Also $G_{1} G G_{2}$ is a straight line. 